Preface

\title{
Thrombosis and Hemostasis in Surgery
}

\author{
Anne-Mette Hvas, MD, $\mathrm{PhD}^{1}$ Julie Brogaard Larsen, $\mathrm{MD}^{1}$ \\ Leonardo Pasalic, PhD, MBBS, FRCPA, FRACP ${ }^{2,3}$ \\ ${ }^{1}$ Centre for Hemophilia and Thrombosis, Department of Clinical \\ Biochemistry, Aarhus University Hospital, Aarhus, Denmark \\ 2 Department of Haematology, Institute of Clinical Pathology and \\ Medical Research, NSW Health Pathology, Westmead Hospital, \\ Sydney, Australia \\ ${ }^{3}$ Sydney Centres for Thrombosis and Haemostasis, \\ Sydney, Australia \\ Semin Thromb Hemost 2017;43:649-652.
}

The present issue of Seminars in Thrombosis \& Hemostasis focuses on challenges related to hemostatic and thromboembolic complications in surgery or trauma. Multiple factors contribute to perioperative bleeding or thromboembolic risk. These factors might be related to the individual patient (e.g., bleeding disorders, cancer, or developmental hemostatic changes in pediatric patients), to anticoagulant medication or complications such as heparin-induced thrombocytopenia (HIT), or to inherent risks of a particular surgical procedure or affected organ (e.g., hepatobiliary surgery or renal transplantation). All of these factors can have a profound effect on expected outcomes.

Curnow provides, as the initial contribution to this issue, an in-depth review of how to manage and support patients with hereditary or acquired bleeding disorders undergoing surgery. ${ }^{1}$ In regard to hereditary bleeding disorders, patients with hemophilia are especially likely to undergo surgery during life due to arthropathy subsequent to repeating joint bleeds. Thorough interdisciplinary planning is crucial to manage the specialized treatment with specific factor concentrates. Curnow also addresses the need for an individualized venous thrombosis risk assessment following surgery, including evaluation of whether thromboprophylaxis is needed and safe in this setting. ${ }^{1}$ Another important aspect comprises pain management in patients with bleeding disorders, as the commonly used analgesic agents can be associated with an increased bleeding risk, and recommendations for handling this are therefore also provided in this review. ${ }^{1}$

The risk of venous thromboembolism is considerably increased in cancer patients, ${ }^{2}$ and as major surgery in itself increases the risk, cancer patients undergoing surgery are particularly prone to development of thromboembolic complications. However, pharmacological thromboprophylaxis is complicated by the fact that cancer patients also have increased risk of bleeding during this treatment. ${ }^{3}$ Exploring these clinical dilemmas, Htun and Lee summarize the evidence on the efficacy and safety of primary thromboprophylaxis in cancer patients undergoing surgery, also taking into account the influence of cancer type on thromboembolic risk; they also present the current consensus recommendations in this setting. ${ }^{4}$

Pediatric cardiac surgery affords special challenges in regard to management of hemostasis. First of all, hemostasis in children differs from that in adults, ${ }^{5}$ making interpretation of laboratory tests challenging. Although several methods exist for hemostatic evaluation, both plasma-based tests and dynamic whole blood coagulation tests, the use of reference values and decision limits for guidance of hemostatic treatment in children are sparsely documented. Ravn therefore summarizes and discusses studies evaluating viscoelastic tests such as ROTEM and TEG as well as platelet function tests in children undergoing cardiac surgery. ${ }^{6}$ Moreover, the review provides valuable information on heparin use and reversal in pediatric cardiopulmonary bypass, and finally, the potential use of antifibrinolytics in the pediatric population is challenged. ${ }^{6}$

A new class of non-vitamin $\mathrm{K}$ oral anticoagulants (NOACs), also called direct oral anticoagulants (DOACs), and comprising direct anti-thrombin and anti-Xa agents, has now become available worldwide and is increasingly being used. One of the advantages of NOACs is that no regular laboratory monitoring is needed, but in case of emergency surgery anticoagulant assessment is warranted. As a result, a
Address for correspondence Anne-Mette Hvas, MD, PhD, Centre for Hemophilia and Thrombosis, Department of Clinical Biochemistry, Aarhus University Hospital, Palle JuulJensens Boulevard 99, DK-8200 Aarhus N, Denmark (e-mail: am.hvas@dadlnet.dk).
Issue Theme Thrombosis and Hemostasis in Surgery; Guest Editors: Anne-Mette Hvas, MD, PhD, Julie Brogaard Larsen, MD, and Leonardo Pasalic, PhD, MBBS, FRCPA, FRACP.
Copyright (c 2017 by Thieme Medical Publishers, Inc., 333 Seventh Avenue, New York, NY 10001, USA.

Tel: +1(212) 584-4662.
DOI https://doi.org/ 10.1055/s-0037-1605571. ISSN 0094-6176. 
variety of laboratory tests have been introduced, and in the present issue of Seminars in Thrombosis \& Hemostasis, Blennerhassett and colleagues review and synthesize the complexities of laboratory measurement of NOAC anticoagulant effects. ${ }^{7}$ Furthermore, the recent advances in development of specific NOAC reversal agents are discussed. The review ends up providing tools for perioperative management of patients taking NOACs based on the current evidence. $^{7}$

Antiplatelet therapy is the cornerstone of primary and secondary prevention of coronary artery disease. ${ }^{8}$ On the other hand, antiplatelet therapy is also associated with an increased risk for perioperative bleeding complications, which is particularly the case in patients treated with acetylsalicylic acid (aspirin) in combination with $\mathrm{P} \mathrm{Y}_{12}$ inhibitors such as clopidogrel. ${ }^{9}$ Perioperative bleeding, with antiplatelet therapy being one of the contributors, has been a serious complication in cardiac surgery ever since its inception, with severe bleeding complications contributing considerably to operative mortality. ${ }^{10}$ To balance the competing needs of reducing thromboembolic complications and minimizing perioperative bleeding, several clinical questions arise, commonly including the timing of discontinuation of antiplatelet therapy, the relevance of platelet function testing prior to surgery, and the optimal time for restarting antiplatelet therapy postoperatively. Hansson and Jeppsson comprehensively address all these issues and provide a summary of evidencebased recommendations on handling antiplatelet therapy in patients undergoing cardiac surgery. ${ }^{11}$

Nearly all patients undergoing cardiopulmonary bypass are exposed to heparin intraoperatively. This heparin exposure is associated with the risk of developing prothrombotic complications of HIT. ${ }^{12}$ Although unfractionated heparin, which carries the highest risk for development of HIT, ${ }^{12}$ is increasingly replaced by low molecular heparin in other clinical settings, HIT remains a threat in cardiac surgery patients. HIT arises in around 1 to $2 \%$ of cardiac surgery patients exposed to heparin, ${ }^{12}$ and if left untreated, the initial rate of thromboembolism, amputation, and death is around $6 \%$ per day. ${ }^{13}$ Thus, obtaining a timely and correct diagnosis is crucial. However, the clinical diagnosis might be challenging because the majority of patients experience a decrease in postoperative platelet count, which can be difficult to distinguish from development of the HIT. Herein, Pishko and Cuker review the epidemiology of HIT after cardiac surgery and provide special insight in the considerations needed regarding both clinical and laboratory diagnosis of HIT. ${ }^{14}$ Finally, the difficult task of anticoagulation in patients with previous HIT requiring future cardiac surgery is addressed. ${ }^{14}$

In surgical patients, the focus has been on pharmacological prophylaxis to reduce the intra- and postoperative thromboembolic risk. Nonetheless, increasing attention is being paid to other modalities such as ischemic conditioning, which has been shown to attenuate ischemia-reperfusion injury. ${ }^{15}$ Hence, this intervention has been suggested as a possible future additional intervention during surgery to prevent ischemia-reperfusion injury. However, the mechanisms behind the tissue-protective effect of ischemic conditioning are still not clarified. The review by Krag and Hvas summarizes the existing evidence of the effects of local or remote ischemic conditioning on laboratory hemostasis measures as well as the incidence of thromboembolism and bleeding in patients undergoing surgery or cardiac procedures. ${ }^{16}$ This systematic review points to the fact that although conditioning consistently reduced platelet activation in patients undergoing cardiac procedures, the intervention did not increase bleeding risk, but notably the majority of studies also indicated that the intervention did not reduce arterial thromboembolic risk in surgery or other cardiac procedures. ${ }^{16}$ Thus, the possible role of ischemic conditioning during surgery still needs further investigations.

Hepatobiliary surgery is associated with an increased risk of both substantial perioperative bleeding and thrombotic complications. Bos and colleagues highlight the complex nature of changes in both primary and secondary hemostasis as well as fibrinolysis in patients with liver disease undergoing hepatobiliary surgery. ${ }^{17}$ The hemorrhagic and thrombotic complications are reviewed and risk factors and possible predictors of hemostatic complications in the perioperative period of hepatobiliary surgery are discussed. Finally, the need for pharmacological thromboprophylaxis following liver surgery is emphasized due to the hypercoagulable postoperative state. ${ }^{17}$ The authors stress the need for further research within this area, as the prevalence of venous thromboembolic events in patients undergoing hepatobiliary surgery continues to be relatively high.

During recent years, more effective immunosuppressive drugs and advances in surgical techniques have led to improvement in patient and graft survival after renal transplantation. ${ }^{18}$ Still, perioperative bleeding and thrombosis affect both patient and graft survival. ${ }^{19}$ Patients with chronic kidney disease, especially those with end-stage renal disease, exhibit hemostatic changes conferring both a prothrombotic state and coagulopathy leading to increased bleeding risk. ${ }^{19,20}$ These hemostatic changes, including the role of thrombophilia, are reviewed by Poli et al, followed by the recommendations for prophylaxis of graft thrombosis and prophylaxis and treatment of venous thromboembolism following renal transplantation. ${ }^{21}$

The case fatality in subarachnoid hemorrhage is high, ${ }^{22}$ and half of the survivors are left with permanent disabilities. ${ }^{23}$ Rebleeding contributes to further increased mortality ${ }^{24}$ and neurosurgery does not prevent all cases of rebleeding. ${ }^{25}$ As subarachnoid hemorrhage has been shown to be associated with increased fibrinolysis, antifibrinolytics could potentially reduce the risk of rebleeding. However, available guidelines differ on this particular issue. ${ }^{26,27}$ In the present issue of Seminars in Thrombosis \& Hemostasis, Anker-Møller et al provide a systematic review on the effect of the use of antifibrinolytics following subarachnoid hemorrhage. ${ }^{28}$ They conclude that tranexamic acid reduced the overall risk of rebleeding following nontraumatic subarachnoid hemorrhage. Moreover, a statistically nonsignificant reduction in mortality was found in both nontraumatic and traumatic subarachnoid hemorrhage, still without substantial indication of increased risk of ischemic lesions. ${ }^{28}$ 
Trauma is one of the leading causes of mortality in the world, and hemorrhage is one of the most frequent causes of death in trauma patients. ${ }^{29}$ As presented by Fries et al, overall two different transfusion strategies exist for management of massive bleeding after trauma: a guided and a nonguided treatment strategy, respectively. First, Fries et al provide a review of different transfusion regimens. ${ }^{30}$ Second, the authors address the ongoing debate about which transfusion strategy is superior by reviewing their effect on mortality in trauma patients. ${ }^{30}$ The current evidence indicates a trend toward lower mortality in studies where guided treatment was used, but the studies published so far are mainly retrospective and show a high degree of heterogeneity. Thus, as concluded by Fries et al, randomized controlled trials are strongly needed to ascertain optimal transfusion approaches in trauma patients. ${ }^{30}$

At least some of the aforementioned challenges could be met if coagulation tests had high predictive values in terms of ability to predict perioperative bleeding. Larsen and Hvas present a comprehensive systematic review on the predictive value of plasma and whole blood coagulation tests as well as platelet function tests. ${ }^{31}$ They conclude that while these laboratory tests are useful in the diagnosis and management of perioperative bleeding, they have only limited ability to predict perioperative bleeding in unselected patients. ${ }^{31}$ Therefore, neither plasma-based coagulation tests nor whole blood tests provide valid predictive information when used for screening in unselected patients prior to surgery and should primarily be used for the management of bleeding patients.

In summary, this issue of Seminars in Thrombosis $\mathcal{E}$ Hemostasis highlights several patient-, drug-, and surgeryrelated factors that influence bleeding and thrombosis risk and thus have to be taken into account in the perioperative setting or trauma. The reviews presented also point to the research needed to further expand the scope and improve the quality of data in this area. We hope you will enjoy reading and discussing the variety of topics presented herein.

\section{References}

1 Curnow J. Managing and supporting surgery in patients with bleeding disorders. Semin Thromb Hemost 2017;43(07):653-671

2 Heit JA, Silverstein MD, Mohr DN, Petterson TM, O'Fallon WM, Melton LJ III. Risk factors for deep vein thrombosis and pulmonary embolism: a population-based case-control study. Arch Intern Med 2000;160(06):809-815

3 Prandoni P, Lensing AW, Piccioli A, et al. Recurrent venous thromboembolism and bleeding complications during anticoagulant treatment in patients with cancer and venous thrombosis. Blood 2002;100(10):3484-3488

4 Htun K, Lee AYY. Thromboprophylaxis in cancer patients undergoing surgery. Semin Thromb Hemost 2017;43(07):672-681

5 Andrew M, Vegh P, Johnston M, Bowker J, Ofosu F, Mitchell L. Maturation of the hemostatic system during childhood. Blood 1992;80(08):1998-2005

6 Ravn HB. Hemostasis in pediatric cardiac surgery. Semin Thromb Hemost 2017;43(07):682-690

7 Blennerhassett R, Favaloro EJ, Pasalic L. Novel (oral) anticoagulant challenges in surgery. Semin Thromb Hemost 2017;43(07): 706-715
8 Lewis HD Jr, Davis JW, Archibald DG, et al. Protective effects of aspirin against acute myocardial infarction and death in men with unstable angina. Results of a Veterans Administration Cooperative Study. N Engl J Med 1983;309(07):396-403

9 Yusuf S, Zhao F, Mehta SR, Chrolavicius S, Tognoni G, Fox KK; Clopidogrel in Unstable Angina to Prevent Recurrent Events Trial Investigators. Effects of clopidogrel in addition to aspirin in patients with acute coronary syndromes without ST-segment elevation. N Engl J Med 2001;345(07):494-502

10 Dyke C, Aronson S, Dietrich W, et al. Universal definition of perioperative bleeding in adult cardiac surgery. J Thorac Cardiovasc Surg 2014;147(05):1458-1463.e1

11 Hansson EC, Jeppsson A. Antiplatelet therapy, platelet function testing, and bleeding complications in cardiac surgery patients. Semin Thromb Hemost 2017;43(07):699-705

12 Pouplard C, May MA, Iochmann S, et al. Antibodies to platelet factor 4-heparin after cardiopulmonary bypass in patients anticoagulated with unfractionated heparin or a low-molecularweight heparin : clinical implications for heparin-induced thrombocytopenia. Circulation 1999;99(19):2530-2536

13 Greinacher A, Eichler P, Lubenow N, Kwasny H, Luz M. Heparininduced thrombocytopenia with thromboembolic complications: meta-analysis of 2 prospective trials to assess the value of parenteral treatment with lepirudin and its therapeutic aPTT range. Blood 2000;96(03):846-851

14 Pishko AM, Cuker A. Heparin-induced thrombocytopenia in cardiac surgery patients. Semin Thromb Hemost 2017;43(07):691-698

15 Bøtker HE, Kharbanda R, Schmidt MR, et al. Remote ischaemic conditioning before hospital admission, as a complement to angioplasty, and effect on myocardial salvage in patients with acute myocardial infarction: a randomised trial. Lancet 2010;375 (9716):727-734

16 Krag AE, Hvas AM. Ischemic conditioning as a hemostatic intervention in surgery and cardiac procedures: a systematic review. Semin Thromb Hemost 2017;43(07):716-731

17 Bos S, Bernal W, Porte R, et al. Hemostatic complications in hepatobiliary surgery. Semin Thromb Hemost 2017;43(07): 732-741

18 Ponticelli C, ed. Thrombotic complications. In: Medical Complications of Kidney Transplantation. Boca Raton, FL: CRC Press Taylor \& Francis Group; 2007:409-415

19 Pavord S, Myers B. Bleeding and thrombotic complications of kidney disease. Blood Rev 2011;25(06):271-278

20 Gangji AS, Sohal AS, Treleaven D, Crowther MA. Bleeding in patients with renal insufficiency: a practical guide to clinical management. Thromb Res 2006;118(03):423-428

21 Poli D, Antonucci E, Zanazzi M. Hemostatic complications in renal transplantation. Semin Thromb Hemost 2017;43(07):742-749

22 Nieuwkamp DJ, Setz LE, Algra A, Linn FH, de Rooij NK, Rinkel GJ. Changes in case fatality of aneurysmal subarachnoid haemorrhage over time, according to age, sex, and region: a metaanalysis. Lancet Neurol 2009;8(07):635-642

23 Hackett ML, Anderson CS; The Australian Cooperative Research on Subarachnoid Hemorrhage Study Group. Health outcomes 1 year after subarachnoid hemorrhage: an international populationbased study. Neurology 2000;55(05):658-662

24 Naidech AM, Janjua N, Kreiter KT, et al. Predictors and impact of aneurysm rebleeding after subarachnoid hemorrhage. Arch Neurol 2005;62(03):410-416

25 Laidlaw JD, Siu KH. Ultra-early surgery for aneurysmal subarachnoid hemorrhage: outcomes for a consecutive series of 391 patients not selected by grade or age. J Neurosurg 2002;97(02): 250-258, discussion 247-249

26 Steiner T, Juvela S, Unterberg A, Jung C, Forsting M, Rinkel G; European Stroke Organization. European Stroke Organization guidelines for the management of intracranial aneurysms and subarachnoid haemorrhage. Cerebrovasc Dis 2013;35(02):93-112 
27 Connolly ES Jr, Rabinstein AA, Carhuapoma JR, et al; American Heart Association Stroke Council; Council on Cardiovascular Radiology and Intervention; Council on Cardiovascular Nursing; Council on Cardiovascular Surgery and Anesthesia; Council on Clinical Cardiology. Guidelines for the management of aneurysmal subarachnoid hemorrhage: a guideline for healthcare professionals from the American Heart Association/American Stroke Association. Stroke 2012;43(06):1711-1737

28 Anker-Møller T, Troldborg A, Hvas AM. Evidence for the use of tranexamic acid in subarachnoid- and subdural hemorrhage: a systematic review. Semin Thromb Hemost 2017;43(07):750-758
29 Kauvar DS, Lefering R, Wade CE. Impact of hemorrhage on trauma outcome: an overview of epidemiology, clinical presentations, and therapeutic considerations. J Trauma 2006;60(6, Suppl): S3-S11

30 Fries D, Innerhofer P, Spahn DR. Transfusion approaches and mortality in trauma patients: a narrative review. Semin Thromb Hemost 2017;43(07):759-771

31 Larsen JB, Hvas AM. Predictive value of whole blood and plasma coagulation tests for intra- and postoperative bleeding risk: a systematic review. Semin Thromb Hemost 2017;43(07): $772-806$ 